Article

\title{
Integration of Multiple Resolution Data in 3D Chromatin Reconstruction Using ChromStruct
}

\author{
Claudia Caudai ${ }^{1, *(D)}$, Monica Zoppè ${ }^{2}$ D , Anna Tonazzini ${ }^{1}\left(\mathbb{D}\right.$, Ivan Merelli ${ }^{3}$ and Emanuele Salerno $^{1}(\mathbb{D}$ \\ 1 National Research Council of Italy, Institute of Information Science and Technologies, 56124 Pisa, Italy ; \\ anna.tonazzini@isti.cnr.it (A.T.); emanuele.salerno@isti.cnr.it (E.S.) \\ 2 National Research Council of Italy, Institute of BioPhysics, 20133 Milano, Italy; monica.zoppe@cnr.it \\ 3 National Research Council of Italy, Institute of Biomedical Technologies, 20054 Segrate, Italy; \\ ivan.merelli@itb.cnr.it \\ * Correspondence: claudia.caudai@isti.cnr.it
}

check for updates

Citation: Caudai, C.; Zoppè, M.; Tonazzini, A.; Merelli, I.; Salerno, E. Integration of Multiple Resolution Data in 3D Chromatin Reconstruction Using ChromStruct. Biology 2021, 10, 338. https://doi.org/

10.3390/biology10040338

Academic Editor: Massimo La Rosa

Received: 1 March 2021

Accepted: 15 April 2021

Published: 16 April 2021

Publisher's Note: MDPI stays neutral with regard to jurisdictional claims in published maps and institutional affiliations.

Copyright: (c) 2021 by the authors. Licensee MDPI, Basel, Switzerland. This article is an open access article distributed under the terms and conditions of the Creative Commons Attribution (CC BY) license (https:// creativecommons.org/licenses/by/ $4.0 /)$.

\begin{abstract}
The three-dimensional structure of chromatin in the cellular nucleus carries important information that is connected to physiological and pathological correlates and dysfunctional cell behaviour. As direct observation is not feasible at present, on one side, several experimental techniques have been developed to provide information on the spatial organization of the DNA in the cell; on the other side, several computational methods have been developed to elaborate experimental data and infer 3D chromatin conformations. The most relevant experimental methods are Chromosome Conformation Capture and its derivatives, chromatin immunoprecipitation and sequencing techniques (CHIP-seq), RNA-seq, fluorescence in situ hybridization (FISH) and other genetic and biochemical techniques. All of them provide important and complementary information that relate to the three-dimensional organization of chromatin. However, these techniques employ very different experimental protocols and provide information that is not easily integrated, due to different contexts and different resolutions. Here, we present an open-source tool, which is an expansion of the previously reported code ChromStruct, for inferring the 3D structure of chromatin that, by exploiting a multilevel approach, allows an easy integration of information derived from different experimental protocols and referred to different resolution levels of the structure, from a few kilobases up to Megabases. Our results show that the introduction of chromatin modelling features related to CTCF CHIA-PET data, histone modification CHIP-seq, and RNA-seq data produce appreciable improvements in ChromStruct's 3D reconstructions, compared to the use of HI-C data alone, at a local level and at a very high resolution.
\end{abstract}

Keywords: chromatin conformation; bayesian statistics; HI-C data; chromatin conformation capture; CTCF CHIA-PET data; CHIP-seq; RNA-seq

\section{Introduction}

The three-dimensional organization of chromatin is involved in regulation of gene function and connected with physiological and pathological correlates and dysfunctional cell behavior. A advancement in chromatin studies was made possible with the development of next-generation sequencing techniques [1], which enabled a number of methods of Chromosome Conformation Capture and its derivatives, such as HI-C techniques [2]. These techniques provide information on how frequently the possible chromatin fragment pairs are in close contact in a population of cells. Depending on the experimental details and on the restriction enzyme used, the genomic resolution of HI-C can range from a few kilobases to Megabases. The opportunity to observe the chromatin structure at multiple resolutions emerged recently, following investigations on the 3D structure of TADs (Topologically Associating Domains) [3], broadly defined as portions of chromatin with more internal than external interactions, are sometimes described as fairly isolated globular 
structures. TADs have a dynamic nature and play a role in gene expression and maintenance of cellular identity [4]. Chromatin conformation is also associated with metabolic activity: for example, transcription requires the DNA to be accessible to a large number of enzymes, involved in all steps: from regulation to intiation, and to progression of the RNA polymerases, often in multiple copies, along the transcribed DNA portion, extending up to many tens of kilobases. This activity, in which the epigenetic code of histone modifications has a role, as reviewed in [5], implies different degrees of chromatin compaction.

In addition to HI-C, other experimental techniques such as CHIP-seq using specific antibodies and RNA-seq can provide information on geometrical features of chromatin [6-10]. CHIP-seq experiments allow the characterization of genomic loci by their association with specific proteins, such as transcription factors or other DNA binding proteins (e.g., CTCF), or by finer molecular details, such as histonic modifications, (e.g., acetylation, mono- or three-methylation at specific histone sites), all of which are associated with distinct functional genomic features. RNA-seq experiments provide information on which DNA loci have been transcribed, identifying genomic portions that are accessible to the transcription machinery, and loose enough to allow exposure of the sequence. Careful consideration of all the information available suggests that some data can (or should) be redundant, while others are complementary or mutually explanatory. In [11], Lieberman Aiden et al. showed, by Principal Component Analysis, that the distribution of contacts in the HI-C matrices correlates with the distribution of genes and with features of open or silent chromatin. For example, expressed genes are frequently marked by a chromatin state that includes H3K27AC modification in their enhancer region and H3K4ME3 in their promoter region, while repressed genes are often marked by the H3K27ME3 modification in their body $[12,13]$. However, not all expressed genes bear such a mark, and not all marked genes are necessarily expressed.

The method proposed here enables the elaboration of plausible 3D chromatin conformations through the integration of several pieces of information. The integration of data at different resolutions permits the derivation of structural properties that are not easily deduced using the different data separately. A number of computational methods have been developed to determine the 3D structures of chromosomes from contact-frequency matrices [14-17]. Many of these methods transform contact frequencies into Euclidean distances, and then reconstruct the structure by solving a distance-to-geometry problem (for example, see [17]). This frequency-to-distance transformation, however, presents a major problem, as it invariably produces geometrically inconsistent distance sets $[18,19]$. To avoid the drawbacks of this strategy, we reject the derivation of distances from the contact frequencies, and adopt an iterative multiscale procedure to derive the 3D structure directly from the contact frequencies. In previous work [20], we introduced ChromStruct, a method to infer a set of spatial chromatin conformations starting from the contact information of HI-C experiments. This method is based on a multiscale chromatin chain model made of consecutive and partially penetrable beads of different sizes. The algorithm automatically divides the contact matrix into variable-size diagonal blocks, and reconstructs the related 3D structures independently for each block. This is made possible by the fact that the chromatin chain presents regions, such as the TADs, with many internal interactions between pairs of loci and interact much less with other regions of the chain. Each diagonal block is used to estimate the structure of the related sub-chain by sampling a solution space generated by a score function based on both data-fit and implicit, soft, geometrical constraints. The sub-chains thus obtained are then modeled as single beads in a coarser-scale chain and the procedure iteratively repeats following the same rules used for the finer scales, until no more isolated blocks are detected in the binned data matrix, i.e., the entire chromosome is modeled. The whole chain is then reconstructed iteratively from the coarsest to the finest scale, by substituting each bead with the corresponding sub-chain at the finer scale, maintaining its 3D orientation (see Figure 1). The intention of our sampling of the solution space is not to find a unique consensus, but a family of solutions. This is consistent with the fact that hi-c data derive from millions of cells, where 
different configurations contribute. The ChromStruct sampling strategy does not search for a global minimum, but explores the solution space to find a number of configurations with similar scores. An advantage of the ChromStruct strategy is that the score function is designed to allow the user to introduce and integrate different features and data sources, even at different resolution levels.

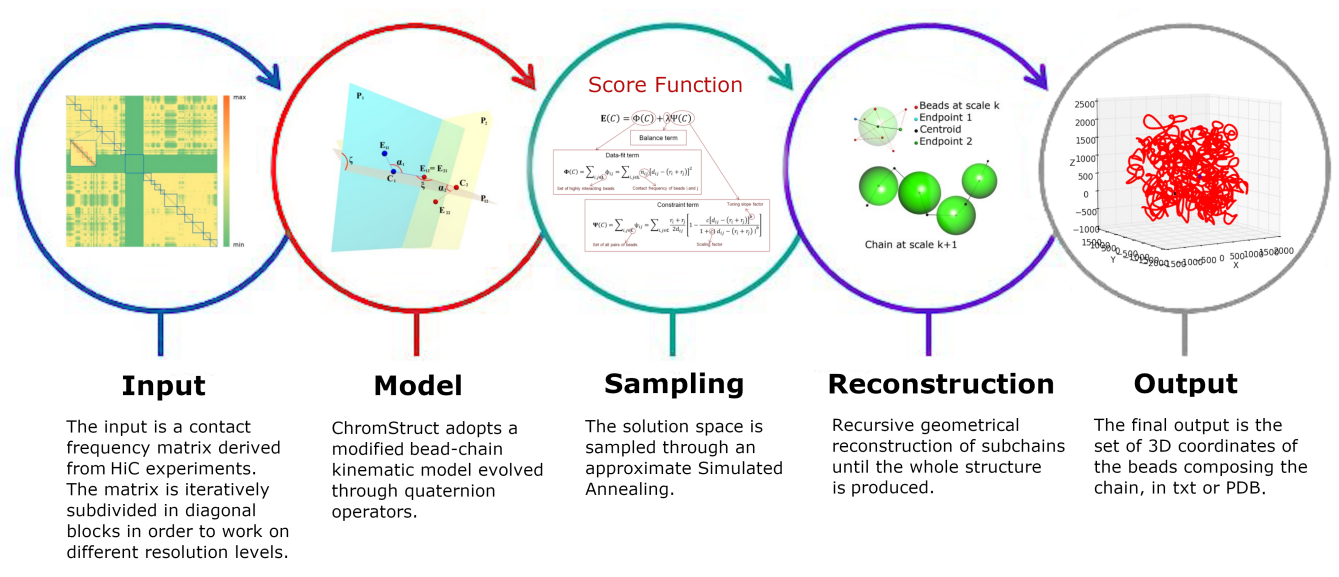

Figure 1. Flow of ChromStruct: (blue) the input HI-C contact frequency matrix is subdivided in diagonal blocks. (red) Chromatin fibre is modeled as a chain of partially penetrable beads and subdivided into sub-chains. (green) Geometrical perturbations are performed in the quaternion algebra and the solution space is sampled by a Bayesian method. (violet) As the last step, a multilevel $3 \mathrm{D}$ reconstruction generates chromatin output conformations (gray) that are compatible with input and constraints.

In this paper, we present an extension of ChromStruct, which allows the HI-C data to be integrated with data derived from other experimental techniques, and demonstrate its use with histone modification specific CHIP-seq, RNA-seq and CTCF CHIA-PET data. The algorithm browses different resolution levels, from the smallest sub-TAD to the entire chromosome, enabling the investigation of the details of folding inside the TADs, at the intermediate structures of nested domains, and at the macroscopic organization of the compartments at the coarsest scale. HI-C experiments provide information on contact frequencies between portions of the chromatin fiber; histone mark CHIP-seq data provide additional information about DNA geometry and 3D occupancy; RNA-seq data provide information about gene expression and, therefore, on the compactness of DNA; finally, CTCF CHIA-PET data inform us about loops that bring distant genome elements into spatial proximity. We introduce these data in the score function as geometrical information. Our results show that the introduction of CTCF CHIA-PET data, RNA-seq and CHIP-seq information can produce more detailed conformations at very high resolutions (few $\mathrm{kb}$ ), while at lower resolution, such improvement is not perceived.

\section{Results}

In this work, we describe an extension of ChromStruct to integrate information derived from $\mathrm{HI}-\mathrm{C}$ experiments with further geometrical data derived from histone specific modification (H3K27ME3) CHIP-seq, RNA-seq and CTCF CHIA-PET experiments. These data are obtained through different laboratory approaches; this is advantageous from a scientific point of view, because they refer to conformational information from different perspectives. For example, portions of DNA showing a high degree of H3K27 three-methylation are enriched in repressed genes and are, therefore, more compact [13], whereas portions where RNA-seq signal the presence of expressed genes are more expanded. A score function that can be interpreted as a log-posterior probability evaluated by applying the Bayes rule manages the relevance of the geometric information available, rewarding the configurations that are consistent with 
the data of different nature and penalizing the ones that appear discordant or uncertain as geometrical interpretations of multiple experimental data. ChromStruct samples the solution space generated by this score function by an approximated simulated annealing [21]. In our experiments, we considered chromosome 12 of human hematopoietic progenitor cells. We collected HI-C contact matrix at a $5 \mathrm{~kb}$ resolution, RNA-seq data, H3K27ME3 CHIP-seq data at 20 bp resolution [8] and CTCF CHIA-PET data [22] (see Section 4 for details). With these data, we compared the ChromStruct's reconstructions using HI-C data alone and using $\mathrm{HI}-\mathrm{C}$ data integrated with H3K27ME3-CHIP-seq, RNA-seq and CTCF CHIA-PET data.

\subsection{High-Resolution Configurations}

ChromStruct takes HI-C contact frequency matrices as the first input. These can be very large (as in the case of chromosome 12 at $5 \mathrm{~kb}$ resolution), and in order to manage the amount of data and lower the computational costs, the first step of the algorithm consists in the division into blocks, respecting the fractal structure of the TADs [21]. The blockdetection algorithm (based on Moving Average on sliding triangles on the main diagonal, described in detail in [21,23]) found 2097 diagonal blocks with an average genomic size of 12 fragments of $5 \mathrm{~kb}$ (i.e., $60 \mathrm{~kb}$ ). To analyze the behavior of the new score-function at a $5 \mathrm{~kb}$ resolution, we selected a smaller $3.5 \mathrm{Mb}$ portion of chromosome 12, containing 50 blocks, and a variety of chromosomal features, as reported in Table 1. As shown, 16 blocks are interested by Histone 3 Lysine 27 three-methylation, 9 by gene expression and in 7 blocks, we have CTCF-mediated internal contacts. Because repression and expression have opposite effects, the score-function annihilates their contribution in the few blocks in which both are contained.

The score function allows the fiber's curvature to be higher in portions of chromatin with H3K27ME3 and penalizes high curvatures in portions interested by expressed genes. The bead diameters also depend on whether they belong to expressed or silent areas (see Section 4 for details). The CTCF feature is modeled as an increase in contact frequency within the HI-C matrix, for the pairs characterised by CTCF-coupling, so as to represent a stronger constraint for their proximity (see Section 4 and Supplementary). Figure 2 shows the comparison between the distributions of correlations between the contact matrices calculated from the estimated configurations and the original $\mathrm{HI}-\mathrm{C}$ contact matrix for the block in the portion of chromosome 12 considered. In the left panel, we consider the blocks interested by active genes, in the central panel the blocks interested by H3K27ME3, associated with repression and, in the right panel, all blocks with CTCF mediated contacts. The boxplots show that, at a $5 \mathrm{~kb}$ resolution, the integration of geometrical information other than HI-C contacts improves the correlation between the synthetic contact matrices and the original contact matrix. The introduction of additional geometrical constraints in our experiments thus allows, at this resolution level, the reconstruction of more accurate high-resolution configurations.

Correlation of Synthetic Contact Matrices and Input Contact Matrix at $5 \mathrm{~kb}$ resolution
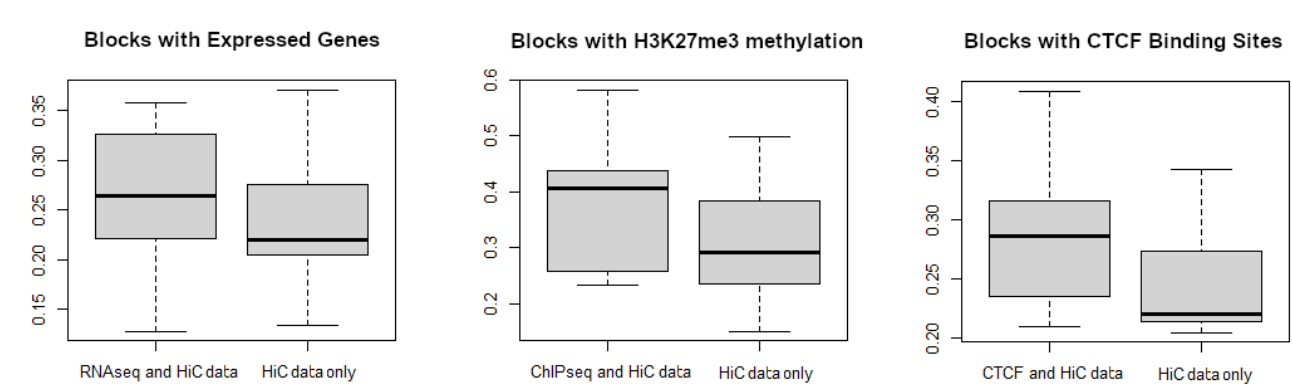

Figure 2. Comparison of distributions of Pearson correlation between contact matrices obtained with ChromStruct and original contact matrix for blocks belonging to a $3.5 \mathrm{Mb}$ portion of chromosome 12 . Blocks interested by expressed genes (left), H3K27ME3 (centre), and CTCF CHIA-PET (right) show a higher correlation if the relevant information is used. 
Table 1. Presence of structural information derived from H3K27ME3 CHIP-seq, RNA-seq and CTCFbinding experiments for $3.5 \mathrm{Mb}$ portion of chromosome 12 [111.5 Mb-115 Mb], corresponding to blocks from 1750 to 1799 identified by block-detection algorithm.

\begin{tabular}{|c|c|c|c|c|c|}
\hline Block & Dimension (kb) & Tot Contacts & Data & Corr $1^{a}$ & Corr $2^{b}$ \\
\hline 1750 & 75 & 272 & Expr genes & 0.128 & 0.134 \\
\hline 1751 & 50 & 102 & Expr genes & 0.489 & 0.428 \\
\hline 1752 & 65 & 295 & Expr genes & 0.246 & 0.191 \\
\hline 1753 & 55 & 142 & Expr genes & 0.264 & 0.217 \\
\hline 1754 & 100 & 133 & Expr genes, CTCF & 0.219 & 0.219 \\
\hline 1755 & 70 & 41 & Expr genes, CTCF & 0.251 & 0.242 \\
\hline 1756 & 65 & 158 & Expr genes & 0.358 & 0.295 \\
\hline 1757 & 85 & 128 & Expr genes, CTCF & 0.286 & 0.217 \\
\hline 1758 & 100 & 211 & Expr genes & 0.222 & 0.204 \\
\hline 1759 & 45 & 89 & Expr genes & 0.320 & 0.371 \\
\hline 1760 & 70 & 247 & Expr genes & 0.325 & 0.341 \\
\hline 1761 & 70 & 153 & & 0.113 & 0.185 \\
\hline 1762 & 50 & 149 & & 0.137 & 0.280 \\
\hline 1763 & 55 & 178 & & 0.322 & 0.364 \\
\hline 1764 & 70 & 168 & & 0.056 & 0.119 \\
\hline 1765 & 100 & 228 & & 0.133 & 0.161 \\
\hline 1766 & 50 & 81 & Expr genes & 0.154 & 0.186 \\
\hline 1767 & 45 & 163 & Expr genes & 0.346 & 0.255 \\
\hline 1768 & 40 & 343 & & 0.164 & 0.201 \\
\hline 1769 & 55 & 268 & & 0.222 & 0.160 \\
\hline 1770 & 50 & 78 & Expr genes, CTCF & 0.326 & 0.204 \\
\hline 1771 & 60 & 38 & Expr genes & 0.178 & 0.244 \\
\hline 1772 & 110 & 389 & Expr genes & 0.303 & 0.235 \\
\hline 1773 & 70 & 90 & & 0.041 & 0.136 \\
\hline 1774 & 100 & 637 & & 0.230 & 0.178 \\
\hline 1775 & 50 & 86 & & 0.184 & 0.163 \\
\hline 1776 & 80 & 383 & Н3К27M3 & 0.233 & 0.236 \\
\hline 1777 & 45 & 77 & H3К27M3 & 0.582 & 0.499 \\
\hline 1778 & 60 & 143 & & 0.306 & 0.318 \\
\hline 1779 & 65 & 179 & CTCF & 0.408 & 0.342 \\
\hline 1780 & 55 & 77 & & 0.428 & 0.443 \\
\hline 1781 & 85 & 66 & CTCF & 0.305 & 0.304 \\
\hline 1782 & 105 & 249 & & 0.324 & 0.241 \\
\hline 1783 & 50 & 39 & & 0.473 & 0.443 \\
\hline 1784 & 85 & 218 & & 0.330 & 0.313 \\
\hline 1785 & 63 & 45 & CTCF & 0.209 & 0.210 \\
\hline 1786 & 70 & 123 & & 0.230 & 0.257 \\
\hline 1787 & 45 & 104 & Н3К27M3 & 0.423 & 0.291 \\
\hline 1788 & 70 & 283 & & 0.145 & 0.131 \\
\hline 1789 & 70 & 142 & & 0.081 & 0.123 \\
\hline 1790 & 45 & 80 & & 0.202 & 0.224 \\
\hline 1791 & 35 & 30 & & 0.220 & 0.258 \\
\hline 1792 & 65 & 185 & Н3К27M3 & 0.425 & 0.392 \\
\hline 1793 & 70 & 208 & H3К27M3 & 0.407 & 0.303 \\
\hline 1794 & 50 & 53 & & -0.05 & 0.014 \\
\hline 1795 & 40 & 168 & H3К27M3 & 0.449 & 0.373 \\
\hline 1796 & 140 & 1659 & Н3К27M3 & 0.250 & 0.286 \\
\hline 1797 & 70 & 240 & H3К27M3 & 0.266 & 0.155 \\
\hline 1798 & 60 & 289 & Н3К27M3 & 0.233 & 0.150 \\
\hline 1799 & 65 & 264 & & 0.141 & 0.063 \\
\hline
\end{tabular}




\subsection{Low-Resolution Configurations}

Our algorithm reconstructs the chains at lower resolutions by binning the blocks at the current resolution level and associating them into single beads in the lower-resolution chain [24] with sizes derived from the three-dimensional structures at the previous level. As shown in Table 2, the fine details at a $5 \mathrm{~kb}$ resolution are not visible at lower resolutions. Indeed, the information related to CHIP-seq and RNA-seq is only detected at the same resolution for which these data are relevant. At lower levels, their introduction no longer highlights significant differences compared to the use of HI-C data only. The correlation between the original $\mathrm{HI}-\mathrm{C}$ contact matrix of the whole chromosome 12 and the synthetic contact matrices obtained by pooling 100 conformations generated by ChromStruct using HI-C data only and 100 conformations generated using HI-C, H3K27ME3-CHIP-seq, RNA-seq and CTCF CHIA-PET data, show no significant differences. A reconstruction of the selected portion of chromosome 12 [111.5 Mb-115 Mb] at a $5 \mathrm{~kb}$ resolution is represented in Figure 3a; a reconstruction of the whole chromosome at $500 \mathrm{~kb}$ resolution is shown in Figure 3b.

Two main reasons can explain the different behaviour at high- and low-resolution. First, the information contained in the HI-C contact matrix and the one derived from histone-mark immuno-precipitation sequencing and RNA-sequencing are not independent. This can be seen in Figure 3c, where the right-hand side, with higher contact density (more yellow in the contact matrix), contains fewer genes (ENCODE tract), and is more interested by Histone 3K27 three-methylation. The opposite is visible in the part on the left. As Lieberman-Aiden et al. demonstrated in [11], HI-C contact matrices already contain a lot of structural information correlated with the distribution of genes and with the features of open chromatin. The second reason is that ChromStruct, in its multi-level approach, already takes into account the existing correlation between contact density and compactness of the chromatin fiber. Specifically, ChromStruct sets the size of every bead in inverse proportion to its number of contacts [21]. Blocks with many contacts reasonably correspond to more compact fiber portions, while blocks with few contacts are likely to correspond to more expanded and more easily transcribed areas. As in large-scale geographical maps, the fine details, such as minor roads or buildings, are not visible but appear when the scale is progressively refined. In our case, the presence of high-resolution details does not affect the overall appearance at large scales, which is completely determined by low-resolution data.

Table 2. Pearson correlations between synthetic contact matrices and original HI-C contact matrix of the whole chromosome 12 for two populations of conformations: using $\mathrm{HI}-\mathrm{C}$ data only (Experiment 1) and using HI-C, CHIP-seq, RNA-seq and CTCF-binding site data (Experiment 2).

\begin{tabular}{|c|c|c|c|c|c|c|}
\hline & HI-C Contacts & RNA-seq & CHIP-seq & CTCF-Binding & Nr of Runs & Correlation $^{a}$ \\
\hline Experiment 1 & $\checkmark$ & & & & 100 & 0.7188371 \\
\hline Experiment 2 & $\checkmark$ & $\checkmark$ & $\checkmark$ & $\checkmark$ & 100 & 0.6963284 \\
\hline
\end{tabular}

From our experiments, we observe that ChromStruct, equipped with the score function described in Section 4, makes it possible to investigate the spatial organization to a high degree of detail, introducing more precise information in the reconstruction at very high resolution $(5 \mathrm{~kb})$. Moving to lower resolutions, these details are not delineated; however, the macroscopic structure and the various dimensions remain consistent with the structural information derived by $\mathrm{HI}-\mathrm{C}$ contact matrices alone. 
a)

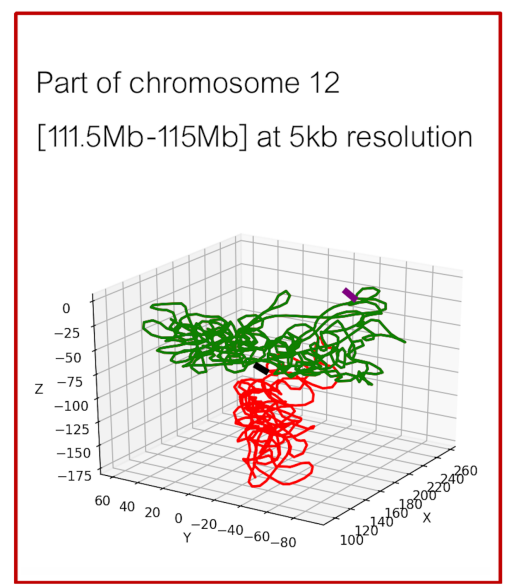

b)

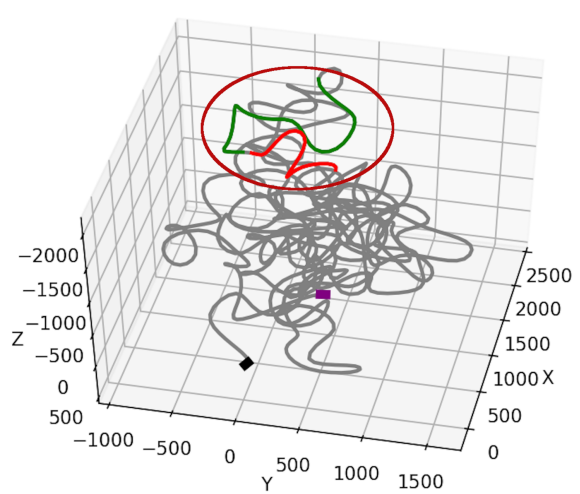

c)

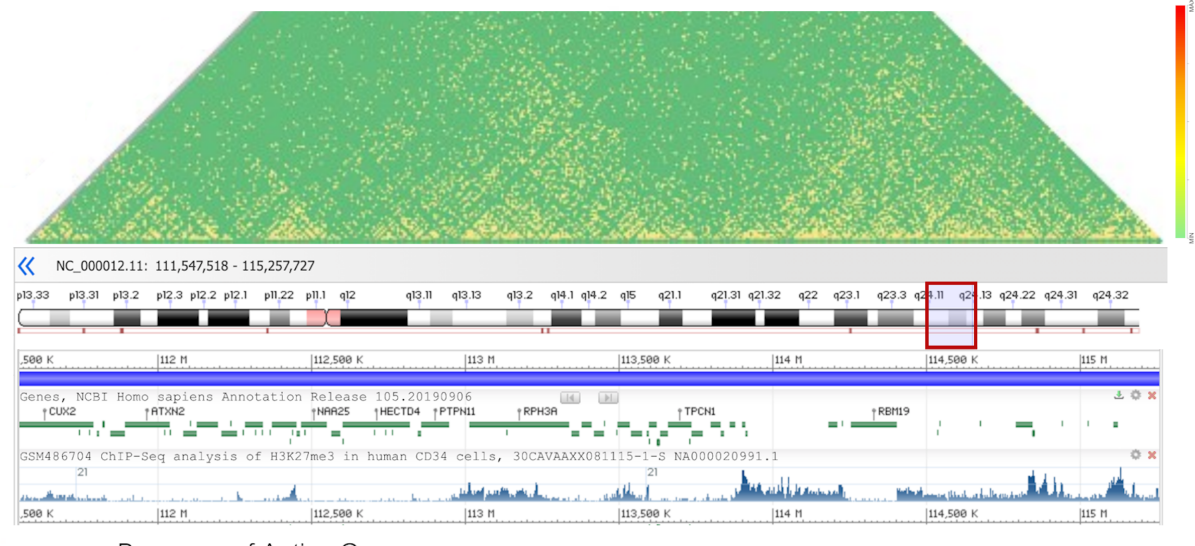

d)

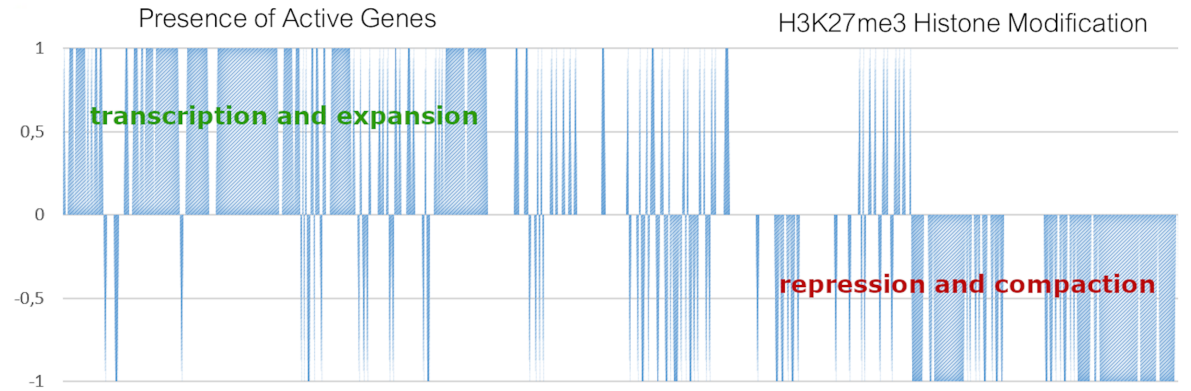

Figure 3. (a) Reconstruction of a portion of chromosome 12 [from $111.5 \mathrm{Mp}$ to $115 \mathrm{Mp}$ ], at a $5 \mathrm{~kb}$ resolution (starting point in black, end point in purple); in green, the part of chromatin interested by active genes and more expanded; in red, the part interested by H3K27ME3, more compact. (b) Reconstruction of the whole chromosome 12 at a $500 \mathrm{~kb}$ resolution: the part in green, interested by active genes, is not only more expanded, but also outermost in the total chromosome. (c) Representation of HI-C, CHIP-seq and RNA-seq data referred to the same portion of chromosome 12 at a $5 \mathrm{~kb}$ resolution (plot from ENCODE). The areas with active genes show a lower concentration of H3K27ME3, while the areas with fewer genes, which are more methylated and more compact, correspond to higher HI-C contact frequencies (more yellow in the contact matrix heatmap). (d) Plot of CHIP-seq and RNA-seq information in ChromStruct's input: 1, -1 or 0 score for every bin associated to expressed genes, H3K27ME3 and none, respectively (see Supplementary for details).

\section{Discussion}

The organization of chromatin at the resolution levels between the wrapping of DNA around histones and the chromosomal domains is not yet completely explained. Experiments of Chromosome Conformation Capture, and in particular those of HI-C type, have contributed to consistent hypotheses on the organization of chromatin within the nucleus. The relationship between the chromatin 3D structure and epigenetic states has been high- 
lighted since the early times of Chromatin 3D studies [3] and has been exploited to confirm the validity of $3 \mathrm{D}$ reconstructions and to derive further information on chromatin biological features [25-28]. However, the introduction of epigenetic information a priori, as a means for the more detailed elaboration of 3D reconstruction, has been attempted only recently [12,29-31]. As many of the experiments on epigenetic features provide information at different levels of resolution, a multilevel approach appears necessary $[15,32]$. The introduction of different information at different resolution levels also tests the correctness of the available data; the Bayesian approach reinforces information that is consistent from a topological point of view and tends to cancel information coming from conflicting inputs. From our experiments, it emerged that the introduction of data related to three-methylation of Histone 3 Lysine 27, gene expression and CTCF-mediated coupling in the score-function of ChromStruct allows the reconstruction of more accurate conformations at a local level, at a resolution of the same order of magnitude as the data introduced.

\section{Materials and Methods}

\subsection{Data Origin and Treatment}

The HI-C, CHIP-seq and RNA-seq data used for the experiment refer to human CD34 hematopoietic progenitor cells (GM12878) [8,33]. Data on CTCF-mediated coupling, obtained through CHIA-PET experiments, were downloaded from GEO accession number GSM1872886. To create contact frequency matrices, fast $q$ data were translated into sam format with the BOWTIE2 program, using the HG19 alignment as reference genome. Through HICEXPLORER, the sam files were first transformed into bam format, and then into Hierarchical Data Format. We used the R package DIFFHIC [34] (choosing Bonferroni correction to lower the False Discovery Rate [35]) to create contact matrices at $5 \mathrm{~kb}$.

In order to introduce information derived from Chromatin IP and RNA-seq, it is necessary to consider the size resolution of these data. Data from RNA sequencing experiments provide information on transcribed genes; these were introduced as a binary 1-dimensional array at a $5 \mathrm{~kb}$ resolution (" 1 " if the bin is interested by expressed genes, " 0 " otherwise). In the case of the Histone 3 Lysine 27 three-methylation mark, which is associated with repressed genes [36], CHIP-seq data are reported at a resolution of 20 base-pairs, making it necessary to bin the data at the resolution of $\mathrm{HI}-\mathrm{C}$ contact matrices $(5 \mathrm{~kb})$. Once binned, they are introduced as a binary 1-dimension array (" 1 " if the binned data point exceeds the threshold of 300, " 0 " otherwise). Finally, CTCF specific capture HI-C precipitation data inform about two DNA segments that are found in close contact. The segments can be at any genomic distance, and can span between TADs; therefore, this information can either be inserted at a $5 \mathrm{~kb}$ resolution, when the two DNA segments lie in the same TAD, or at a lower resolution, when the DNA segments involved belong to different TADs. CTCF CHIA-PET contact features are introduced as a binary matrix, at the same dimension and same resolution of the $\mathrm{HI}-\mathrm{C}$ contact matrix (" 1 " if the couple of beads correspond to a binding site, " 0 " otherwise). This matrix, multiplied by a scalar factor and added to the HI-C contact matrix, enforces the proximity constraints for binding sites (further details in Supplementary). Features are modelled as binary 1- and 2-dimensional arrays in order to be easily introduced into the score-function; however, other modelling approaches are possible, perhaps weighing features distributions.

\subsection{Volume Considerations}

The interphase nucleus of a typical cell is a roundish structure of about 5 micrometers in diameter. The fraction of the volume actually occupied by chromatin (DNA + histone octamers) is about one third. Historical histological observations describe Eu- and Heterochromatin as two distinct states; recent CRYOEM studies [37] have further characterized this distinction, and shown a Chromatin Volume Concentration (CVC) that ranges from $12 \%$ to $50 \%$. From these values, and following the distribution reported, we can attribute a CVC of $20 \%$ to euchromatin (the transcriptionally active portion of DNA) and of $40 \%$ to heterochromatin (the silent, repressed portion). Based on these considerations, we reduced 
the bead diameters by $10 \%$ in repressed portions and increased them by $10 \%$ in active portions. Our score-function is also designed to modulate the density of CVC to plus or minus $50 \%$, respectively, for regions that contain expressed genes and silent regions, by decreasing and increasing the admissible curvature of the thread.

\subsection{Solution Space Sampling}

The score function that generates the solution space for each sub-chain $\mathcal{C}$ to be estimated has the following form:

$$
\Xi(\mathcal{C})=\Phi_{H i C}(\mathcal{C})+\mu_{1} \Phi_{C h I P}(\mathcal{C})+\mu_{2} \Phi_{R N A}(\mathcal{C})+\lambda \Psi(\mathcal{C})
$$

where $\Phi_{H i C}, \Phi_{C h I P}$ and $\Phi_{R N A}$ are the data-fit terms corresponding to HI-C contact data, CHIP-seq data and RNA-seq data, respectively, $\Psi$ instead is the constraint term. Parameters $\mu_{1}, \mu_{2}$ and $\lambda$ are intended to balance the mutual influence of the different terms.

The term $\Phi_{H i C}$ forces bead pairs with many mutual contacts to be close to each other:

$$
\Phi_{H i C}(\mathcal{C})=\sum_{i, j \in \mathcal{L}} n_{i j}\left[d_{i j}-\left(r_{i}+r_{j}\right)\right]^{2}
$$

where $n_{i j}$ is the contact frequency of the $i$-th and $j$-th beads, $d_{i j}$ is the distance between their centroids, and $r_{i}$ and $r_{j}$ are their radii. $\mathcal{L}$ is a subset of contacts in each sub-chain, made of the pairs exceeding a pre-defined percentile of the contact frequencies in the related block.

By controlling the maximum distance between the beads in the sub-chain, the term $\Phi_{C h I P}$ increases the curvature of a sub-chain affected by methylation. Due to this term, regions with high concentration of Histone modification H3K27ME3 (associated to repression) are steered to be more compact:

$$
\Phi_{\text {ChIP }}(\mathcal{C})=\left[\max _{i, j \in \mathcal{L}_{\mathcal{C H I P}}}\left(d_{i j}\right)-d_{\min }\right]^{2}
$$

where $\mathcal{L}_{\mathcal{C H I P}}$ is the set of all pairs (with the exception of the pairs located on the two main diagonals) if the block is interested by H3K27ME3, the empty set otherwise. The value $d_{\text {min }}$ is derived as follows:

$$
d_{\min }(C)=d_{c} \frac{\sqrt[5 / 6]{R I S}}{6 \pi}
$$

and represents the estimate of the minimum size that a bead can assume at the resolution of RIS (in our case $5 \mathrm{~kb}$ ) with the diameter of the chromatin fiber equal to $d_{c}$ (in our case $30 \mathrm{~nm}$ ). The term $\Phi_{C h I P}$ gives little penalty if the maximum distance between the beads of a sub-chain is close to $d_{\text {min }}$.

The term $\Phi_{R N A}$ acts by controlling the minimum distance between non-adjacent beads in the subchain, thus reducing its curvature and makes regions with active genes more expanded:

$$
\Phi_{R N A}(\mathcal{C})=\left[\min _{i, j \in \mathcal{L}_{\mathcal{R N A}}}\left(d_{i j}\right)-d_{\max }\right]^{2}
$$

where $\mathcal{L}_{\mathcal{R} \mathcal{N A}}$ is the set of all pairs if the block is interested by expressed genes, and the empty set if not. The value $d_{\max }$ represents the estimate of the maximum size for a bead at the resolution of RIS and with a chromatin's diameter of $d_{c}$ :

$$
d_{\max }(C)=d_{c} \frac{\sqrt[5 / 6]{R I S}}{3 \pi}
$$

The term $\Phi_{R N A}$ gives little penalty if the minimum distance between the beads of a sub-chain (except for consecutive ones) is at least $d_{\max }$.

When any two beads in $\mathcal{C}$ interpenetrate, one of the terms in brackets becomes negative. The maximum data-fit penalisation of this situation occurs when $d_{i j}=0$, and in Equation (2) 
$\varphi_{i j}$ assumes the finite and unmodifiable value $n_{i j}\left(r_{i}+r_{j}\right)^{2}$. The constraint term $\Psi$ is needed to control this penalisation:

$$
\Psi(\mathcal{C})=\sum_{i, j \in \mathcal{C}} \frac{r_{i}+r_{j}}{2 d_{i j}}\left[1-\frac{\left\{c\left[d_{i j}-\left(r_{i}+r_{j}\right)\right]\right\}^{b}}{1+\left\{c\left|d_{i j}-\left(r_{i}+r_{j}\right)\right|\right\}^{b}}\right]
$$

where $c$ is a scale factor that makes the terms in braces dimensionless, and the exponent $b$ is an odd natural. For $d_{i j}$ near zero, $\psi_{i j}$ behaves as $\left(r_{i}+r_{j}\right) / d_{i j}$, whereas in an interval around $\left(r_{i}+r_{j}\right)$ it behaves as $\left(r_{i}+r_{j}\right) /\left(2 d_{i j}\right)$ and, for $d_{i j}$ sufficiently larger than $\left(r_{i}+r_{j}\right)$, it goes rapidly to zero. Parameter $b$ tunes the slope of the transitions between the different zones; large values of $b$ produce abrupt transitions. Term $\Psi$ is intended to prevent any two beads from interpenetrating more than some fraction of their sizes. Note that, when adjacent or genomically close beads are involved, modulating the allowed mutual interpenetration is also effective to avoid knots and to constrain the local curvature of the chain.

In order to allow easy setting of the geometric parameters related to the data resolution, the size of the beads, the diameter of the fibre, the mechanism of subdivision into TADs and the coefficients of the score function, ChromStruct is equipped with a Graphical User Interface (GUI). The GUI, illustrated in Figure 4, also allows users to insert the input data from dialog boxes: the HI-C matrix (required) and the CHIP-seq, RNA-seq and CTCFbinding arrays (optional). Guidelines for GUI's use are detailed in the Supplementary Materials.

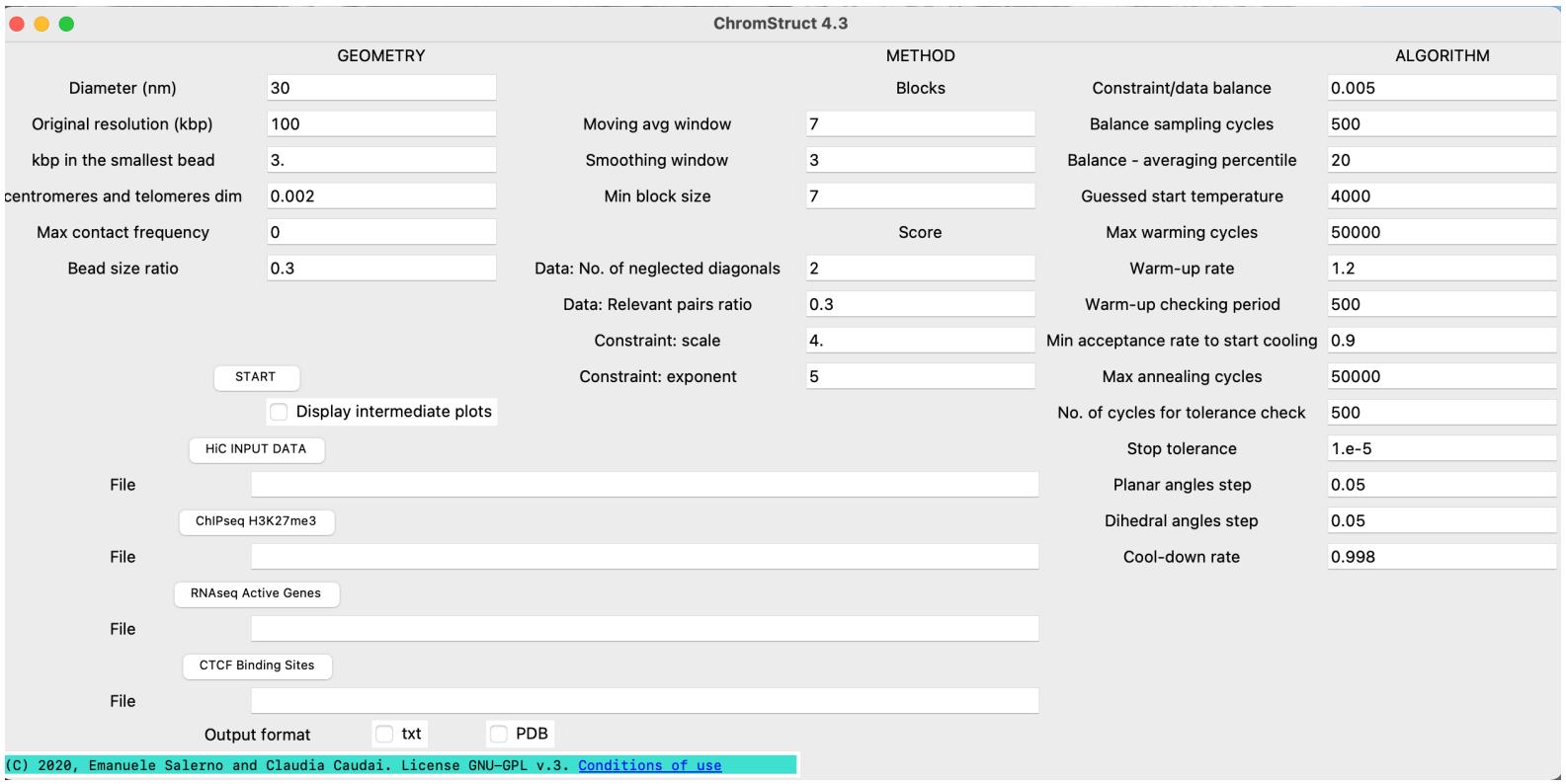

Figure 4. Graphical User Interface of ChromStruct. Three groups of quantities are displayed: the first (GEOMETRY) includes geometrical features, the second (METHOD) sets up the TADs extraction and the score function, and the third (ALGORITHM) is only related to the Simulated Annealing parameters.

\section{Conclusions}

Using multilevel approaches in computational biology is, in some cases, necessary: on the one hand, this strategy makes it possible to parallelise the algorithms, greatly reducing the computational cost. On the other hand, it becomes possible to introduce data obtained from experiments that produce results at different scales, and to analyse biological structures navigating between different scales.

We have made ChromStruct suitable for introducing information at different resolutions, so as to be able to exploit and integrate as much knowledge as possible on the three-dimensional organization of chromatin, also deriving from different biological experiments and also belonging to different dimensional scales. 
Supplementary Materials: The following are available online at https:/ / www.mdpi.com/2079-773 7/10/4/338/s1.

Author Contributions: conceptualization, C.C., E.S., A.T., I.M. and M.Z.; methodology, C.C. and E.S.; software, C.C., E.S. and I.M.; validation, C.C. and M.Z.; formal analysis, C.C. and E.S.; writing, C.C.; review and editing, E.S. and M.Z.; funding acquisition, A.T.; supervision E.S. and M.Z. All authors have read and agreed to the published version of the manuscript.

Funding: This work has been partially supported by the Italian Flagship Project InterOmics, WP01ISTI, and by ISTI-CNR, through scientific agreement 4249/2017 with ITB-CNR, Milan.

Institutional Review Board Statement: Not applicable.

Informed Consent Statement: Not applicable.

Data Availability Statement: All the data used are publicly available from the URLs mentioned in the paper.

Acknowledgments: The authors thank Luciano Milanesi and Clelia Peano for helpful discussions.

Conflicts of Interest: The authors declare no conflict of interest.

Code Availability: The latest version of ChromStruct code: CHROMSTRUCT V4.3, is available in the Supplementary Materials. Previous versions are publicly available: CHROMSTRUCT V4.2 DOI:10.13140/rg.2.2.26123.39208. chromstruct v3.1 DOI:10.13140/rg.2.2.35785.13923.

\section{References}

1. Schuster, S. Next-generation sequencing transforms today's biology. Nat. Methods 2008, 5, 16-18. [CrossRef]

2. van Berkum, N.L.; Lieberman-Aiden, E.; Williams, L.; Imakaev, M.; Gnirke, A.; Mirny, L.A.; Dekker, J.; Lander, E.S. Hi-C: A Method to Study the Three-dimensional Architecture of Genomes. J. Vis. Exp. 2010, 39, e1869. [CrossRef]

3. Dixon, J.R.; Selvaraj, S.; Yue, F.; Kim, A.; Li, Y.; Shen, Y.; Hu, M.; Liu, J.S.; Ren, B. Topological domains in mammalian genomes identified by analysis of chromatin interactions. Nature 2012, 485, 376-380. [CrossRef] [PubMed]

4. Wang, S.; Su, J.H.; Beliveau, B.J.; Bintu, B.; Moffitt, J.R.; Wu, C.T.; Zhuang, X. Spatial organization of chromatin domains and compartments in single chromosomes. Science 2016, 353, 598-602. [CrossRef] [PubMed]

5. Bártová, E.; Krejcí, J.; Harnicarová, A.; Galiová, G.; Kozubek, S. Histone Modifications and Nuclear Architecture: A Review. J. Histochem. Cytochem. 2008, 56, 711-721. [CrossRef] [PubMed]

6. Marti-Renom, M.A.; Mirny, L.A. Bridging the Resolution Gap in Structural Modeling of 3D Genome Organization. PLoS Comput. Biol. 2011, 7, e1002125. [CrossRef]

7. Romano, O.; Peano, C.; Tagliazucchi, G.M.; Petiti, L.; Poletti, V.; Cocchiarella, F.; Rizzi, E.; Severgnini, M.; Cavazza, A.; Rossi, C.; et al. Transcriptional, epigenetic and retroviral signatures identify regulatory regions involved in hematopoietic lineage commitment. Sci. Rep. 2016, 6, 24724. [CrossRef] [PubMed]

8. Mifsud, B.; Tavares-Cadete, F.; Young, A.C.; Sugar, R.; Schoenfelder, S.; Ferreira, L.; Wingett, S.; Andrews, S.; Grey, W.; Ewels, P.; et al. Mapping long-range promoter contacts in human cells with high-resolution capture Hi-C. Nat. Genet. 2015, 47, 598-606. [CrossRef]

9. Javierre, B.M.; Burren, O.S.; Wilder, S.P.; Kreuzhuber, R.; Hill, S.M.; Sewitz, S.; Cairns, J.; Wingett, S.W.; Várnai, C.; Thiecke, M.J.; et al. Lineage-Specific Genome Architecture Links Enhancers and Non-coding Disease Variants to Target Gene Promoters. Cell 2016, 167, 1369-1384.e19. [CrossRef]

10. Sefer, E.; Kingsford, C. Semi-nonparametric modeling of topological domain formation from epigenetic data. Algorithms Mol. Biol. 2019, 14, 4. [CrossRef]

11. Lieberman-Aiden, E.; van Berkum, N.L.; Williams, L.; Imakaev, M.; Ragoczy, T.; Telling, A.; Amit, I.; Lajoie, B.R.; Sabo, P.J.; Dorschner, M.O.; et al. Comprehensive Mapping of Long-Range Interactions Reveals Folding Principles of the Human Genome. Science 2009, 326, 289-293. [CrossRef]

12. Kuksa, P.P.; Amlie-Wolf, A.; Hwang, Y.C.; Valladares, O.; Gregory, B.D.; Wang, L.S. HIPPIE2: A method for fine-scale identification of physically interacting chromatin regions. NAR Genom. Bioinform. 2020, 2, lqaa022. [CrossRef] [PubMed]

13. Barski, A.; Cuddapah, S.; Cui, K.; Roh, T.; Schones, D.E.; Wang, Z.; Wei, G.; Chepelev, I.; Zhao, K. High-Resolution Profiling of Histone Methylations in the Human Genome. Cell 2007, 129, 823-837. [CrossRef] [PubMed]

14. Varoquaux, N.; Ay, F.; Noble, W.S.; Vert, J.P. A statistical approach for inferring the 3D structure of the genome. Bioinformatics 2014, 30, i26-i33. [CrossRef] [PubMed]

15. Nowotny, J.; Ahmed, S.; Xu, L.; Oluwadare, O.; Chen, H.; Hensley, N.; Trieu, T.; Cao, R.; Cheng, J. Iterative reconstruction of three-dimensional models of human chromosomes from chromosomal contact data. BMC Bioinform. 2015, 16, 338. [CrossRef] [PubMed]

16. Hu, M.; Deng, K.; Qin, Z.; Dixon, J.; Selvaraj, S.; Fang, J.; Ren, B.; Liu, J.S. Bayesian Inference of Spatial Organizations of Chromosomes. PLoS Comput. Biol. 2013, 9, e1002893. [CrossRef] 
17. Rousseau, M.; Fraser, J.; Ferraiuolo, M.A.; Dostie, J.; Blanchette, M. Three-dimensional modeling of chromatin structure from interaction frequency data using Markov chain Monte Carlo sampling. BMC Bioinform. 2011, 12, 414. [CrossRef]

18. Caudai, C.; Salerno, E.; Zoppè, M.; Tonazzini, A. A statistical approach to infer 3D chomatin structure. In Mathematical Models in Biology; Zazzu, V., Ed.; Springer International Publishing: Cham, Switzerland, 2015; pp. 161-171.

19. Duggal, G.; Patro, R.; Sefer, E.; Wang, H.; Filippova, D.; Khuller, S.; Kingsford, C. Resolving spatial inconsistencies in chromosome conformation measurements. Algorithms Mol. Biol. 2013, 8, 8. [CrossRef] [PubMed]

20. Caudai, C.; Salerno, E.; Zoppè, M.; Tonazzini, A. Estimation of the Spatial Chromatin Structure Based on a Multiresolution Bead-Chain Model. IEEE ACM Trans. Comput. Biol. Bioinform. 2019, 16, 550-559. [CrossRef]

21. Caudai, C.; Salerno, E.; Zoppè, M.; Merelli, I.; Tonazzini, A. ChromStruct 4: A Python Code to Estimate the Chromatin Structure from Hi-C Data. IEEE ACM Trans. Comput. Biol. Bioinform. 2018, 16, 1867-1878. [CrossRef]

22. Li, G.; Fullwood, M.; Xu, H.; Mulawadi, F.; Velkov, S.; Vega, V.; Ariyaratne, P.; Mohamed, Y.B.; Ooi, H.S.; Tennakoon, C.; et al. ChIA-PET tool for comprehensive chromatin interaction analysis with paired-end tag sequencing. Genome Biol. 2009, 11, R22. [CrossRef]

23. Lajoie, B.; Dekker, J.; Kaplan, N. The Hitchhiker's guide to Hi-C analysis: Practical guidelines. Methods 2015, 72, 65-75. [CrossRef]

24. Caudai, C.; Salerno, E.; Zoppè, M.; Tonazzini, A. Inferring 3D chromatin structure using a multiscale approach based on quaternions. BMC Bioinform. 2015, 16, 234. [CrossRef] [PubMed]

25. Serra, F.; Baù, D.; Goodstadt, M.; Castillo, D.; Filion, G.; Martí-Renom, M.A. Automatic analysis and 3D-modelling of Hi-C data using TADbit reveals structural features of the fly chromatin colors. PLoS Comput. Biol. 2017, 13, e1005665. [CrossRef] [PubMed]

26. Xie, W.J.; Meng, L.; Liu, S.; Zhang, L.; Cai, X.; Gao, Y. Structural Modeling of Chromatin Integrates Genome Features and Reveals Chromosome Folding Principle. Sci. Rep. 2017, 7, 1-11. [CrossRef] [PubMed]

27. Giorgetti, L.; Galupa, R.; Nora, E.; Piolot, T.; Lam, F.; Dekker, J.; Tiana, G.; Heard, E. Predictive Polymer Modeling Reveals Coupled Fluctuations in Chromosome Conformation and Transcription. Cell 2014, 157, 950-963. [CrossRef]

28. Imakaev, M.; Fudenberg, G.; McCord, R.; Naumova, N.; Goloborodko, A.; Lajoie, B.; Dekker, J.; Mirny, L. Iterative Correction of Hi-C Data Reveals Hallmarks of Chromosome Organization. Nat. Methods 2012, 9, 999-1003. [CrossRef]

29. Abbas, A.; He, X.; Niu, J.; Zhou, B.; Zhu, G.; Ma, T.; Song, J.; Gao, J.; Zhang, M.Q.; Zeng, J. Integrating Hi-C and FISH data for modeling of the 3D organization of chromosomes. Nat. Commun. 2019, 10, 2049. [CrossRef]

30. Paulsen, J.; Sekelja, M.; Oldenburg, A.R.; Barateau, A.; Briand, N.; Delbarre, E.; Shah, A.; Sørensen, A.L.; Vigouroux, C.; Buendia, B.; et al. Chrom3D: Three-dimensional genome modeling from Hi-C and nuclear lamin-genome contacts. Genome Biol. 2017, 18, 21. [CrossRef]

31. Qi, Y.; Zhang, B. Predicting three-dimensional genome organization with chromatin states. PLoS Comput. Biol. 2019, 15, e1007024. [CrossRef]

32. Trieu, T. Oluwadare, O.; Cheng, J. Hierarchical Reconstruction of High-Resolution 3D Models of Large Chromosomes. Sci. Rep. 2019, 9, 1-12. [CrossRef] [PubMed]

33. ENCODE Consortium. An Integrated Encyclopedia of DNA Elements in the Human Genome. Nature 2012, 489, 57-74. [CrossRef]

34. Lun, A.T.L.; Smyth, G. diffHic: A Bioconductor package to detect differential genomic interactions in Hi-C data. BMC Bioinform. 2015, 16, 1-11. [CrossRef]

35. Simes, R. An improved Bonferroni procedure for multiple tests of significance. Biometrika 1986, 73, 751-754. [CrossRef]

36. Tie, F.; Banerjee, R.; Stratton, C.A.; Prasad-Sinha, J.; Stepanik, V.; Zlobin, A.; Diaz, M.O.; Scacheri, P.C.; Harte, P.J. CBP-mediated acetylation of histone H3 lysine 27 antagonizes Drosophila Polycomb silencing. Development 2009, 136, 3131-3141. [CrossRef] [PubMed]

37. Ou, H.D.; Phan, S.; Deerinck, T.J.; Thor, A.; Ellisman, M.H.; O'Shea, C.C. ChromEMT: Visualizing 3D chromatin structure and compaction in interphase and mitotic cells. Science 2017, 357, eaag0025. [CrossRef] [PubMed] 